Biomass derived dialdehyde cellulose crosslinked chitosan based nanocomposite hydrogel with phyto-synthesised zinc oxide nanoparticles for enhanced curcumin delivery and bioactivity

Dhanya George, Palanisamy Uma Maheswari, Khadar Mohamed Meera Sheriffa Begum*, Gangasalam Arthanareeswaran Department of Chemical Engineering, National Institute of Technology, Tiruchirapalli 620015, Tamilnadu, India

\title{
*For Correspondence
}

Dr. K. M. Meera Sheriffa Begum

Professor

Department of Chemical Engineering,

National Institute of Technology - Tiruchirapalli

Tamilnadu, India

E-mail: meera.nitt.edu@gmail.com

Tel.: +91 431 2503109, Fax: +91 4312500133 


\section{Supplementary Files}

Fig. S1. Characterisation of green ZnO NPs: (a) UV-Vis spectroscopy, (b) Zeta potential, (c) FE-SEM and (d) EDX

Fig. S2. The TGA graphs of various carriers

Fig. S3. Signal to Noise ratio plot (Taguchi method)

Fig. S4. Kinetic models for curcumin release from nanocomposite hydrogel and pure chitosan - cellulose hydrogel at various $\mathrm{pH}$

Fig. S5. Kinetic models for drug release from nanocomposite hydrogel and pure chitosan cellulose hydrogel at various curcumin concentrations

Fig. S6. Antibacterial (Staphylococcus aureus) and antifungal (Trichophyton rubrum) activities of the $\mathrm{CHG}, \mathrm{CHG}-\mathrm{ZnO}$, CHG-Cur and $\mathrm{CHG}-\mathrm{ZnO}-\mathrm{Cur}$ systems

Fig. S7 Phase inverted microscopy images of A431 cells at different concentration of Cur, ZnO NPs, CHG-ZnO and CHG-ZnO-Cur 
Figure. S1.

(a)

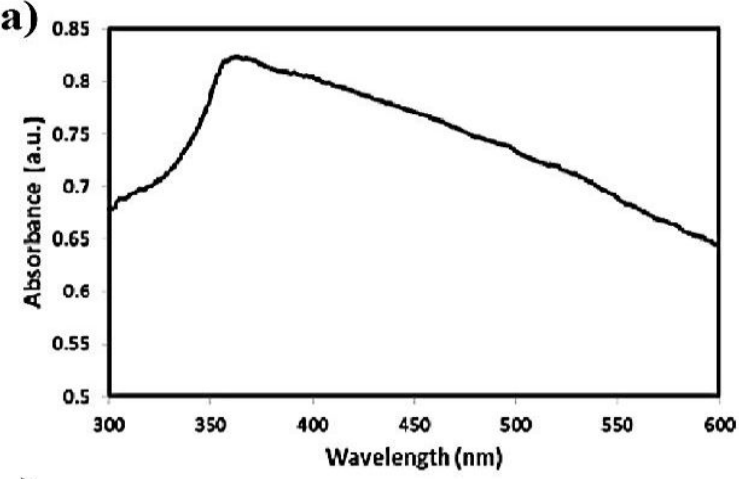

(c)

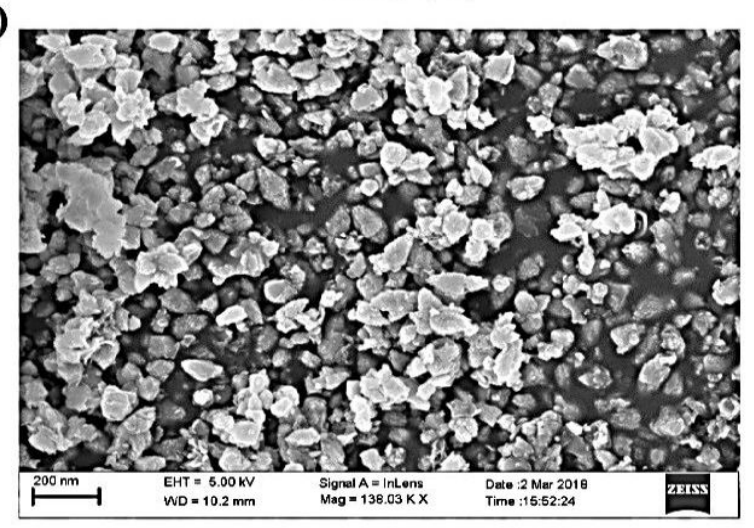

(b)

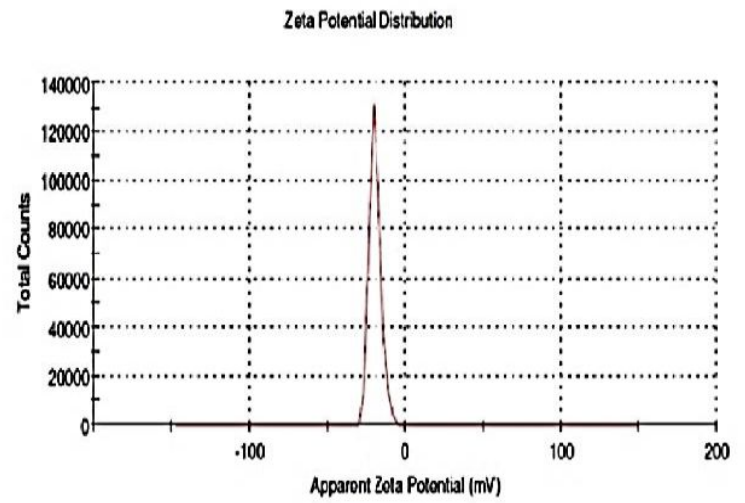

(d)

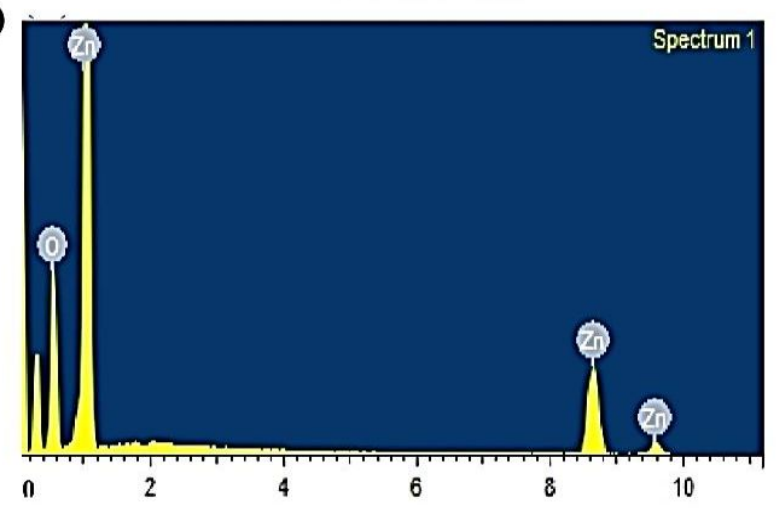


Figure S2

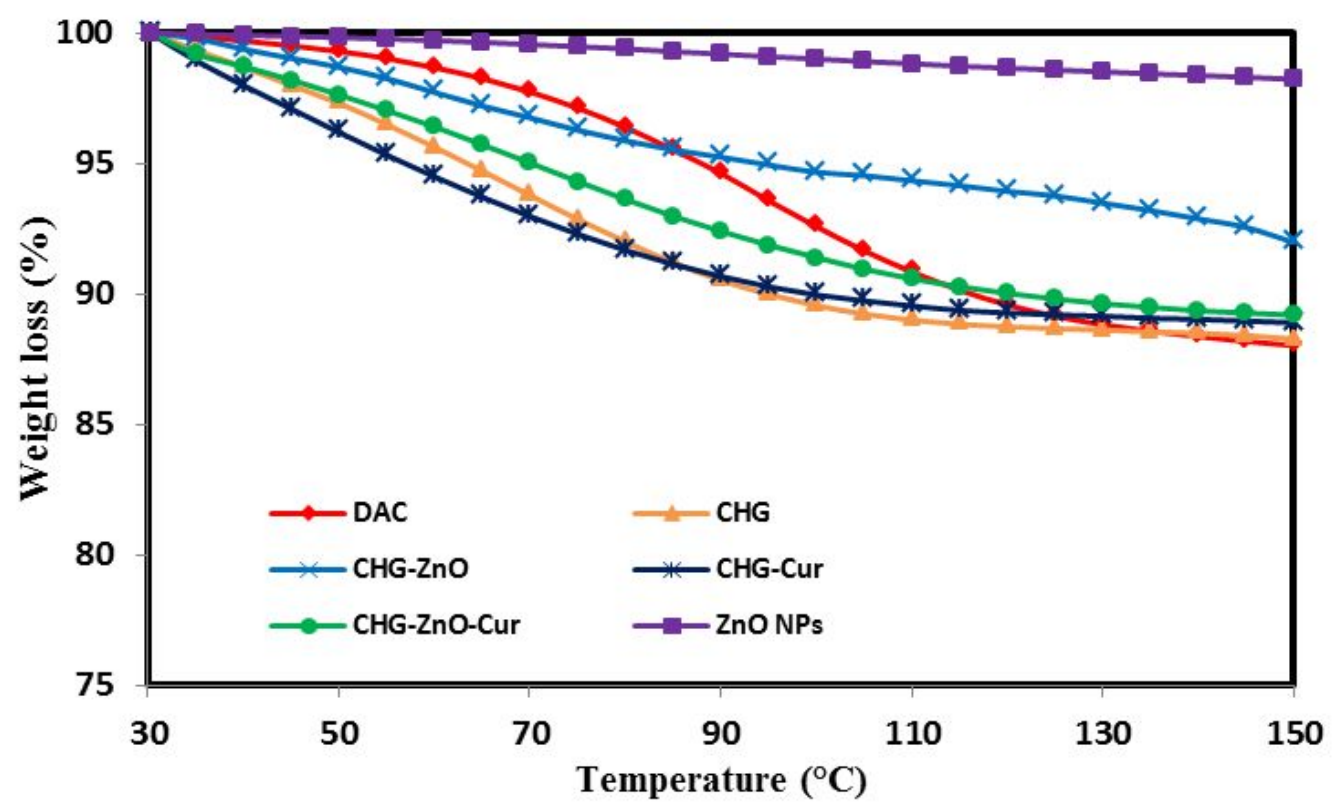


Figure. S3.

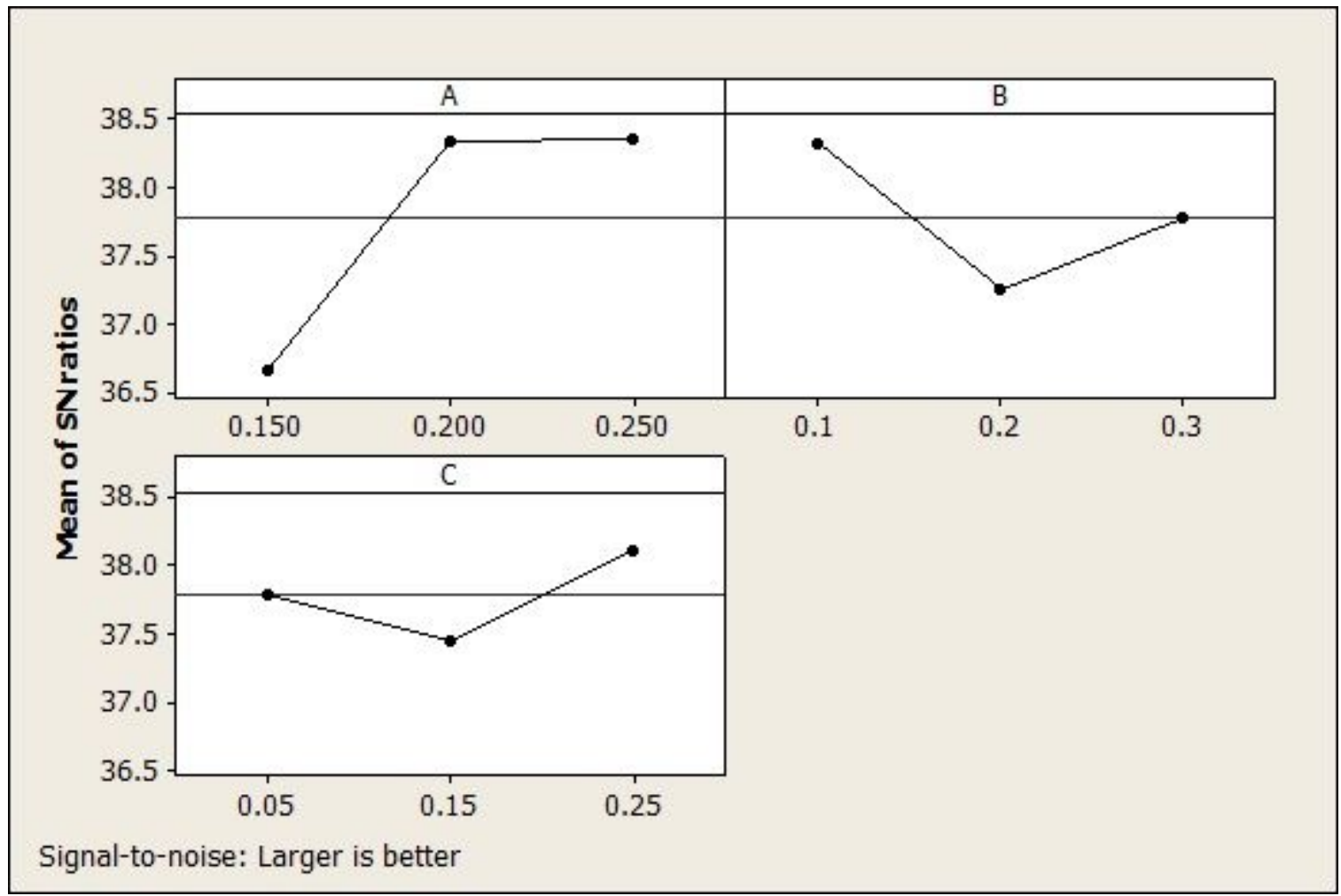


Figure. S4.
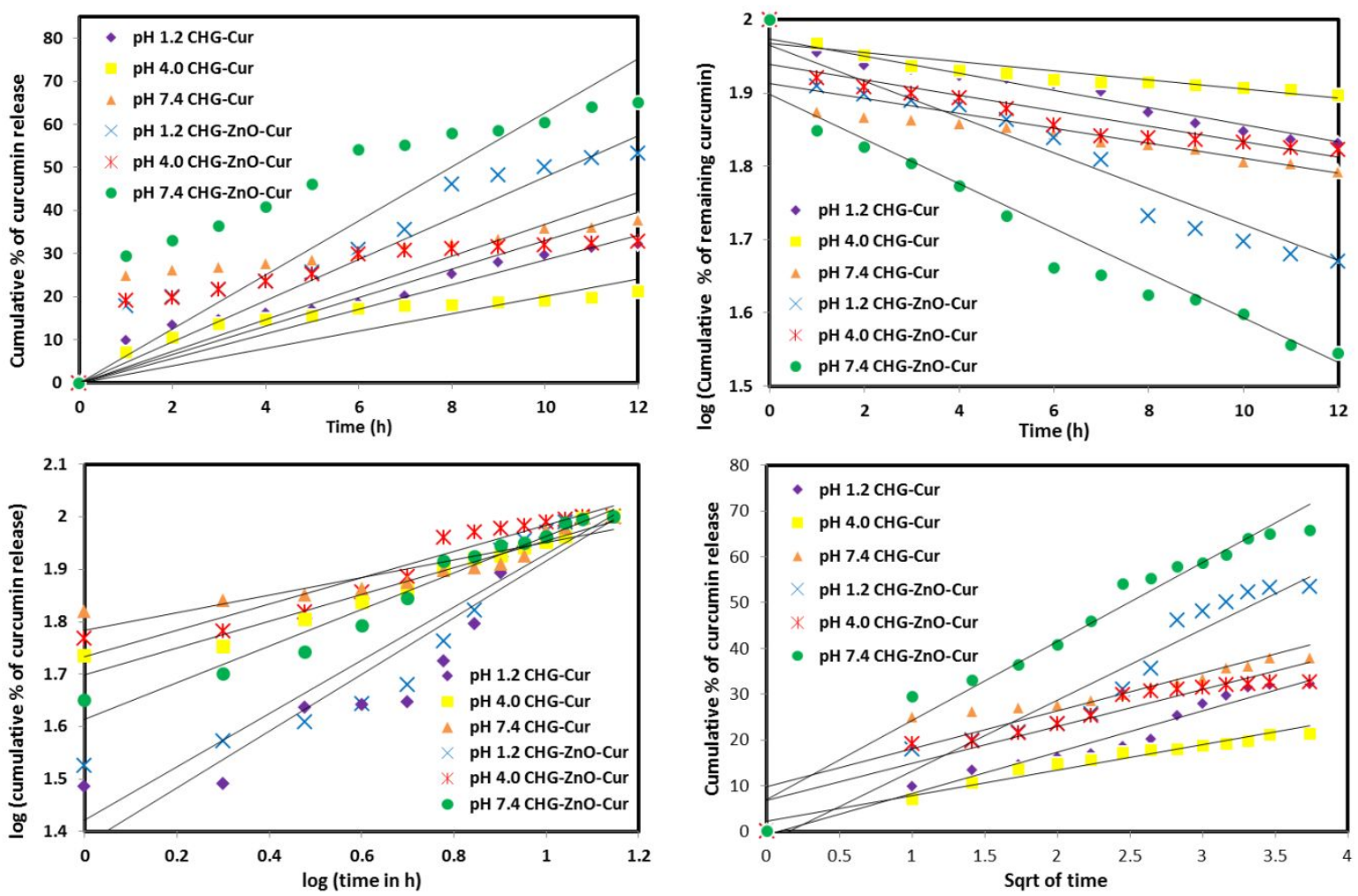
Figure. S5.
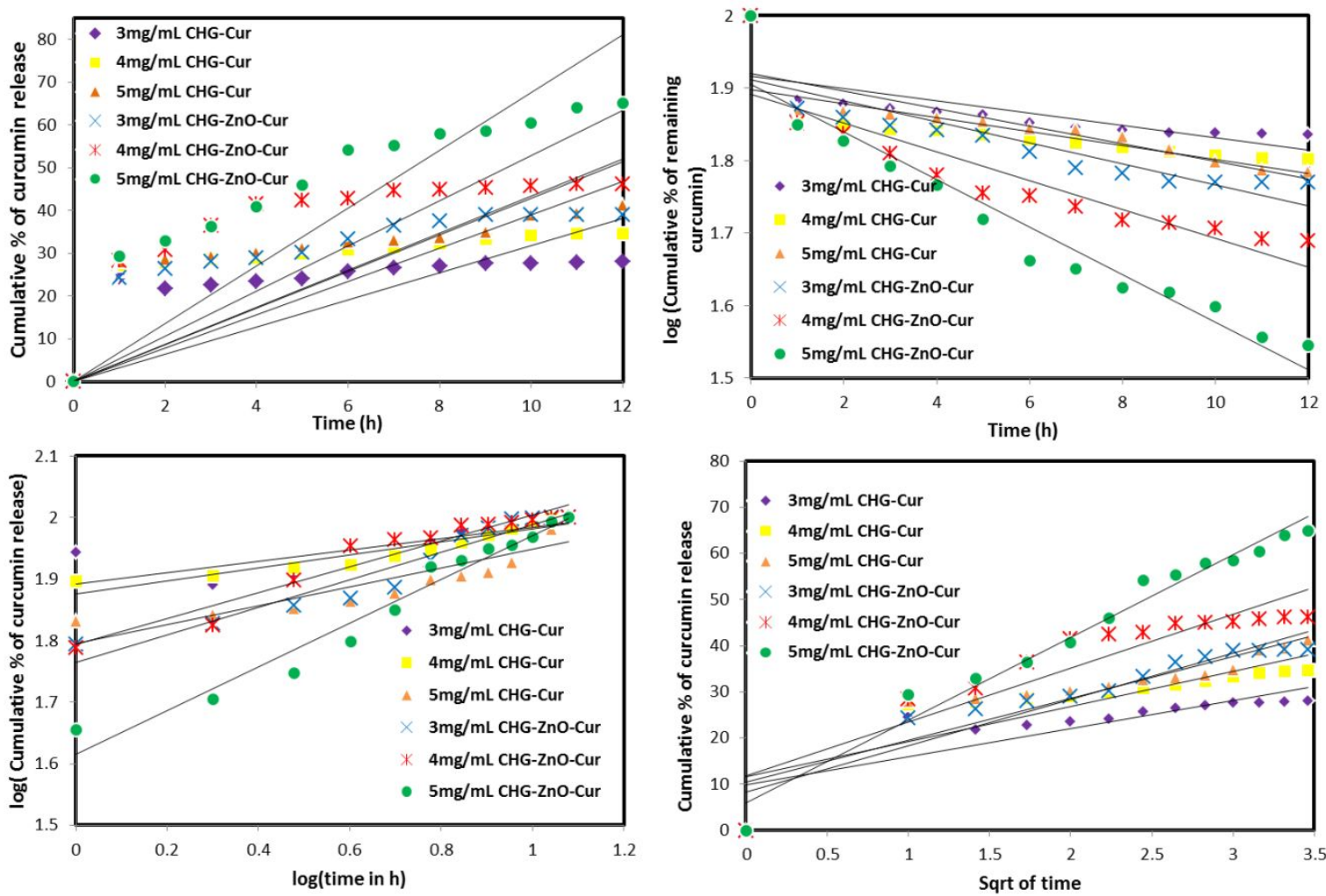
Figure. S6.
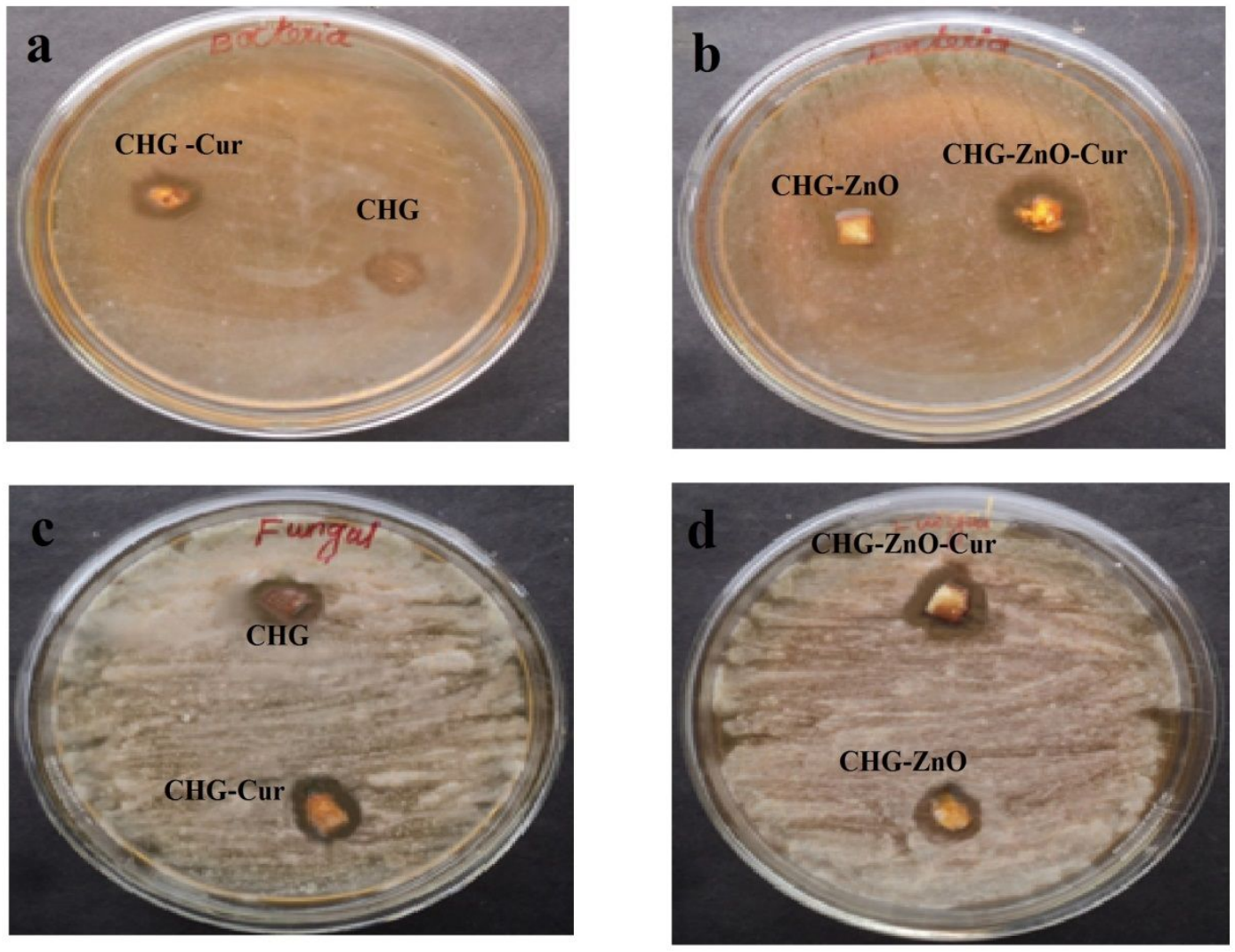
Figure. S7.

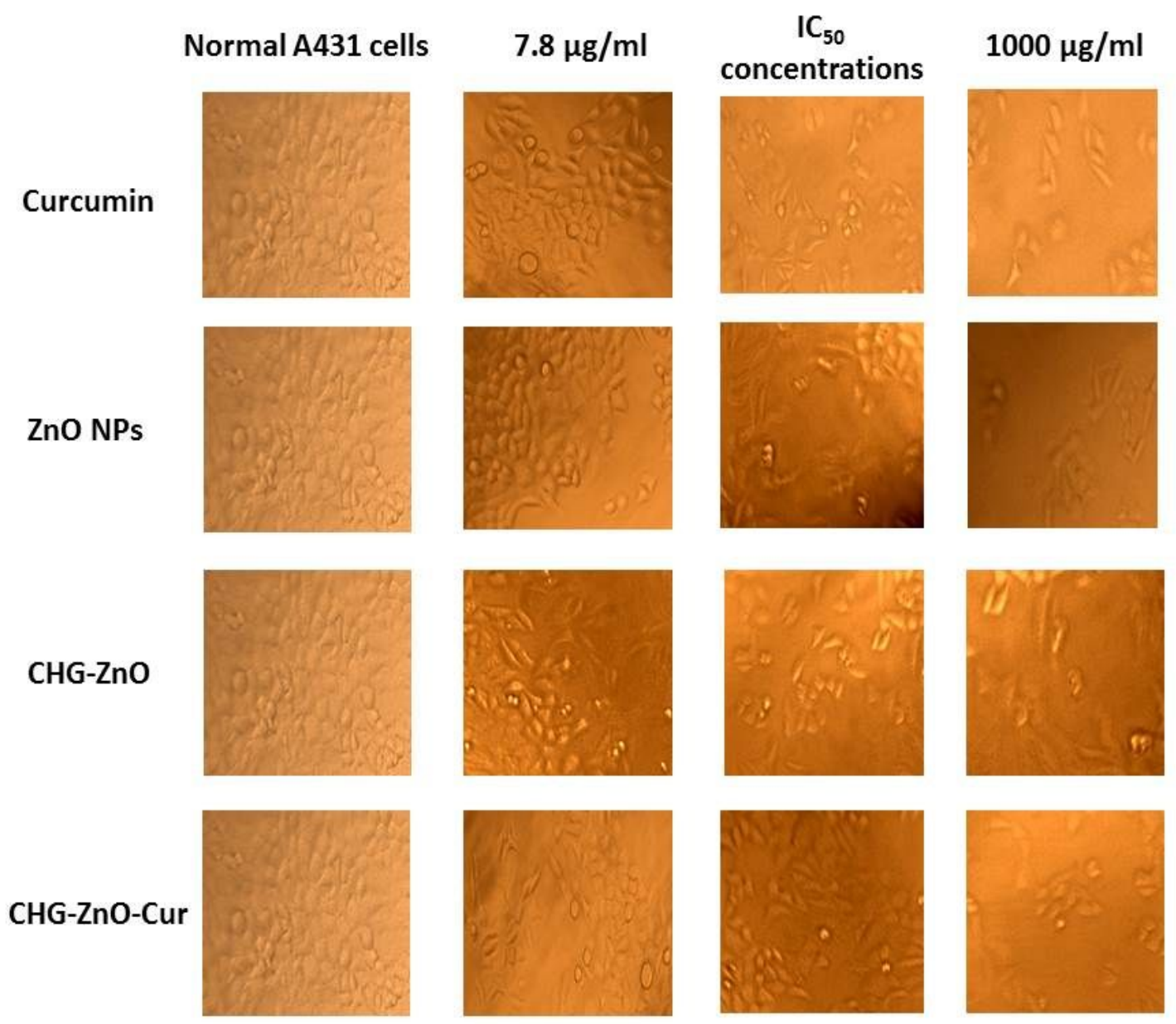


Table S1. ANOVA data analysis

\begin{tabular}{cccccccc}
\hline Variables & $\begin{array}{c}\text { Degree } \\
\text { of } \\
\text { freedom }\end{array}$ & $\begin{array}{c}\text { Sum } \\
\text { of } \\
\text { square }\end{array}$ & Variance & $\begin{array}{c}\text { F } \\
\text { Value }\end{array}$ & $\begin{array}{c}\text { P } \\
\text { Value }\end{array}$ & $\begin{array}{c}\% \\
\text { Contribution }\end{array}$ & Rank \\
\hline A & 2 & 420.361 & 210.181 & 29.22 & 0.033 & 68.997 & 1 \\
B & 2 & 128.174 & 64.087 & 8.91 & 0.101 & 21.038 & 2 \\
C & 2 & 46.232 & 23.162 & 3.22 & 0.237 & 7.588 & 3 \\
Error & 2 & 14.385 & 7.192 & & & & \\
Total & 8 & 609.243 & & & & & \\
\hline
\end{tabular}


Table S2. Kinetic parameters obtained from models on curcumin release from nanocomposite hydrogel at various $\mathrm{pH}$

\begin{tabular}{ccccccc}
\hline Hydrogel & $\mathrm{pH}$ & $\begin{array}{c}\text { Zero Order } \\
\mathrm{R}^{2}\end{array}$ & $\begin{array}{c}\text { First Order } \\
\mathrm{R}^{2}\end{array}$ & $\begin{array}{c}\text { Korsmeyer }- \text { Peppas } \\
\mathrm{R}^{2}\end{array}$ & $\begin{array}{c}\text { Higuchi } \\
\mathrm{n}\end{array}$ & $\mathrm{R}^{2}$ \\
\hline \multirow{3}{*}{ CHG-ZnO-Cur } & 1.2 & 0.125 & 0.941 & 0.9886 & 0.5073 & 0.9431 \\
& 4.0 & 0.8187 & 0.8086 & 0.9749 & 0.3497 & 0.8924 \\
& 7.4 & 0.3585 & 0.9171 & 0.975 & 0.2511 & 0.9653 \\
& 1.2 & 0.792 & 0.9461 & 0.8992 & 0.5409 & 0.9637 \\
CHG-Cur & 4.0 & 0.3386 & 0.8198 & 0.9633 & 0.2548 & 0.9609 \\
& 7.4 & 0.452 & 0.7099 & 0.9456 & 0.1683 & 0.9375 \\
\hline
\end{tabular}


Table S3. Kinetic parameters obtained from models on curcumin release from nanocomposite hydrogel at various curcumin concentrations

\begin{tabular}{ccccccc}
\hline Hydrogel & $\begin{array}{c}\text { Concentration } \\
(\mathrm{mg} / \mathrm{mL})\end{array}$ & $\begin{array}{c}\text { Zero Order } \\
\mathrm{R}^{2}\end{array}$ & $\begin{array}{c}\text { First Order } \\
\mathrm{R}^{2}\end{array}$ & \multicolumn{2}{c}{ Korsmeyer - Peppas } & \multicolumn{2}{c}{ Higuchi } \\
& 3 & 0.6884 & 0.7649 & 0.9739 & 0.2112 & 0.8893 \\
CHG-ZnO-Cur & 4 & 0.5885 & 0.7984 & 0.9629 & 0.2233 & 0.8549 \\
& 5 & 0.8366 & 0.9279 & 0.9817 & 0.3548 & 0.9724 \\
& 3 & 0.5254 & 0.5686 & 0.9650 & 0.1066 & 0.7712 \\
CHG-Cur & 4 & 0.4781 & 0.5374 & 0.9017 & 0.1391 & 0.7194 \\
& 5 & 0.6078 & 0.737 & 0.8973 & 0.1731 & 0.8094 \\
\hline
\end{tabular}

